\title{
Organic Chemical
}

National Cancer Institute

\section{Source}

National Cancer Institute. Organic Chemical. NCI Thesaurus. Code C718.

Molecule that contains atoms of the element carbon, usually combined with itself and with atoms of one or more other element such as hydrogen, oxygen, nitrogen, sulfur, phosphorus, chlorine, or fluorine. Excludes carbides, carbonates and carbon oxides. 